\title{
Collaboration, Dedication, and Social Pressure: A Comparative Analysis of Virtual and Face-to-Face Game Jams
}

\author{
Lingyuan Li \\ Clemson University \\ lingyu2@g.clemson.edu
}

\author{
Divine Maloney \\ Clemson University \\ divinem@g.clemson.edu
}

\author{
Guo Freeman \\ Clemson University \\ guof@clemson.edu
}

\begin{abstract}
Game jams are virtual or face-to-face events where game developers (often non-professional or hobbyist) collaborate to make digital games within a limited time frame. As these events have become not only game-creation events but also social collaboration spaces, important questions emerge regarding how exactly different modes of game jams can offer different affordances for developers' social collaboration. Using 24 semi-structured interviews, we provide a comparative analysis of virtual and face-to-face game jams, especially with regard to the different technological processes and social engagement in each mode of game jams. We contribute to the game jam community by highlighting game jams as novel social collaboration spaces and by providing empirical evidence of the unique affordances of different modes of game jams for social collaboration.
\end{abstract}

\section{Introduction}

Game jams refer to intense and time-sensitive game creation events where a digital game is developed in a relatively short time frame (typically 48 to 72 hours) exploring given design constraints, and end results are shared publicly [1]. They can also be conducted exclusively online through computer-mediated methods (i.e., virtual game jams) or face-to-face. The earliest game jam can be dated back to 2002. Video game developers Chris Hecker and Sean Barrett invited a group of famous designers and programmers at their office to develop games using a newly built game engine [2]. The first virtual game jam "Ludum Dare" was held to invite participants to create a game based on a given theme in the following month. Winners were then determined based on various judging standards through this game jam's online forum [3].

Following this tradition of social collaboration and innovation, nowadays, both virtual and face-to-face game jams have increasingly become popular sites where non-professional game developers (e.g., independent [indie] game developers), amateurs, and hobbyists collaboratively design and develop more creative and novel digital games. As previous studies have shown, they have emerged as not only game-creation events but also as social collaboration spaces where game developers are motivated to meet other people who make games $[4,5]$. Therefore, how exactly different modes of game jams (e.g., virtual vs. face-to-face) can offer different affordances for developers' social collaboration becomes an important research question. Answers to this question would not only benefit the game jam community but also help make game development more open, accessible, and inclusive by shedding light on the social and technological dynamics embedded in game jams regardless of their modes.

To explore this research question, in this paper, we provide a comparative analysis of virtual and face-to-face game jams based on 24 interviews of game jam participants, especially with regard to the different technological processes and social engagement in each mode of game jams. We focus on comparing virtual game jams and face-to-face game jams because virtual game jams have become popular alternatives for collaboratively engaging in game development by reducing costs and attracting broader participants and audiences [6, 7]. Yet still little is known regarding how virtual game jams provide game developers with unique means of preparation, dedication, and social engagement compared to face-to-face jams. We also focus on game jams that aim at indie game developers or hobbyist because they are considered an emerging bottom-up workforce to innovate game development by emphasizing social interaction and community building outside the mainstream gaming industry $[8,9,10,11]$.

We contribute to the game jam community by highlighting game jams as novel social collaboration spaces that emphasize time sensitive and intensive teamwork, more complex and multidimensional interaction dynamics, and more challenging and 
promising community engagement. In addition, We provide empirical evidence of the unique affordances of different modes of game jams for social collaboration, such as the values of networking and the interpersonal aspects of game development in face-to-face game jams and flexibility, freedom, and accessibility in virtual game jams. In a broader sense, these insights on the social and technological dynamics embedded in game jams would also help us better understand the increasingly important computer-mediated collaboration in today's society.

\section{Game Jams and Game Development}

Game jams are described as accelerated opportunistic game creation events that a game is created in a relatively short time frame and with the end results being shared publicly [1]. These events often attract a variety of participants and audiences ranging from hobbyists, indie developers, industrial employees, and academics $[12,1]$. They generally take place as either face-to-face physical jams where participants are physically co-located or virtual jams where participants use computer-mediated methods to collaborate, socialize and co-create.

Prior literature has highlighted several social and technological characteristics of game jams $[13,5,14$, 15]. For example, from a social perspective, game jams allow for open participation that encourages everyone to contribute to game development collaboratively. They require participants to focus game development on $a$ specific thematic topic under time constraints typically range from 48-72 hours. They emphasize teamwork and encourage participants to work together in small teams despite solo game jams exist. In addition, most game jams involve a public presentation component games developed during the time period will be shared with other participants and the general public. From a technological perspective, game jams also demonstrate the focus on mini game prototype that encourages the creation of small, experimental, and playable games as well as tool agnosticism, which promotes the use of a wide variety of tools for developing the prototype. Therefore, game jams have been considered important venues for game developers to collaboratively and rapidly prototype new and disruptive product ideas [7, 16, 4, 17], facilitate openness and participation in game development, and foster a safe, welcoming, and supportive social environment [1, 16, 18, 19].

In this sense, game jams have become not only game-creation events but also important social collaboration spaces, especially for indie and amateur developers. Prior literature has explored similar social collaboration spaces such as fablabs (i.e., fabrication laboratories that focus on design and engineering [20]), hackathons (i.e., events where computer programmers collaborate intensively over a short period of time to develop a software project [21]), and makerspaces (i.e., public workshops where makers can share tools and knowledge [22]). Game jams draw characteristics of all of the three as they focus on designing and development, involve short projects in general, and facilitate collaborative creation-based learning [16, 18].

However, how different modes of game jams (e.g., virtual vs. face-to-face) may offer different affordances for developers' social collaboration and provide unique means of preparation, dedication, and social engagement has not been extensively investigated. Previous studies have shown that similar virtual and physical spaces for social collaboration tend to show different benefits. For example, the collocation of teammates and in-person social coding in offline hackathons would increase interpersonal communication, facilitate better collaboration [23], and promote retention, motivation, and inspiration [24]. Online hackathons, in contrast, would attract a more diverse set of participants and audiences in a particular region and expose them to a variety of thinking and development methodologies [25]. In addition, online hackathons tend to focus on commitment, work quality, and community significance while offline hackathons emphasize accidental complexity, duplicated code, and long methods [26]. How, if at all, do virtual and face-to-face game jams also afford different technological processes, social experiences, and values that are important to game design and development? This concern, therefore, leads to our comparative analysis of virtual and face-to-face game jams.

\section{Methodology}

To recruit participants, we posted a message on Reddit and multiple Discord channels for game developers to recruit interviewees who had participated in any game jam. We also directly contacted game developers in the USA who we already knew and asked their willingness to participate in using a snowball sampling. All developers who responded to our requests and agreed to participate were interviewed. As a result, 24 semi-structured in-depth interviews were conducted via text/audio chat based on participants' modality preferences from September 2019 to November 2019. The average length of interviews was 60 minutes, and participants were given a $\$ 20$ gift card after they completed the interviews.

In each interview, open-ended questions regarding 
developers' experiences and attitudes towards face-to-face and/or virtual game jams were asked. Examples of interview questions included: "What challenges have you faced when participating in face-to-face or virtual game jams? How did you solve them?" "How did you support each other in face-to face or virtual game jams?" and "What strategies, methods, or platforms do you think would facilitate your participation in face-to-face or virtual game jams and innovation?"

Among the 24 participants, three self-identified as female, one as gender non-binary, and twenty as male. Participants aged from 18 to 51 years with an average age of 29.3 years. Eight of them $(33.3 \%)$ developed indie games full-time, while $16(66.7 \%)$ as part-time or a hobby. Participants were located in different countries, including the USA, Canada, India, Malaysia, South Korea, Australia, Nigeria, Brazil, Russia, Japan, the UK, Belgium, and Austria.

We used an empirical, in-depth qualitative analysis of the collected data [27] to investigate game developers' experiences of virtual and face-to-face game jams. All three authors are experienced game researchers, and one of the authors has been studying indie game development and has participated in several local game jams since 2017. Accordingly, we have the knowledge and expertise to study the game jam community. Our analytical procedures were: 1) we closely read through the collected data to acquire a general understanding of the whole picture as regards developers' social collaboration experiences in virtual and face-to-face game jams and collectively identified thematic topics and common features in the data (e.g., team formation; preparations; social interaction; coordination and strategies) for further analysis; 2) we carefully examined and reviewed the thematic topics and developed sub-themes; 3) we collaborated in an iterative coding process to discuss, combine, and refine themes and features to generate a detailed description of comparing social collaboration in virtual and face-to-face game jams.

\section{Findings}

In this section, we present our findings as two parts: 1) shared experiences across virtual and face-to-face game jams, including the need to be prepared in terms of skills, tools, and software installation; the preference for familiarity when teaming; and the sense of challenges due to struggles with time management and scope of work; and 2) unique aspects of social collaboration in virtual and face-to-face game jams, including flexibility, social pressure, and interaction.

\subsection{Shared Experiences in Both Virtual and Face-to-Face Game Jams}

Virtual and face-to-face game jams are essentially the same: game developers need to create a game based on the assigned theme within a certain period of time. Therefore, participants highlighted several similar aspects of their experiences regardless of engaging in virtual or face-to-face game jams, for instance, how they prepared, formed a team, and faced challenges.

Preparation for Required Skills and Tools. We found that participants would do almost the same preparations regardless of the mode of the game jam. Due to the level of intensity of such events, many participants often took time to sharpen their technical skills beforehand in order to be more proficient and effective when developing a game in a time-sensitive manner. P6 (27, non-binary, South Korea, hobbyist) explained:

"No matter online or offline, the jam is often intended to be over a weekend so you have to create everything within two days. So all I really did was grind my technical skill to hopefully make a game that didn't look like absolute doodoo." (P6)

In doing so, participants often relied on refreshing or reinforcing past experiences to get their skills ready, as P12 (23, male, Nigeria, full-time indie) mentioned: "I often get myself ready by watching clips of video of the past jam... And also try to remake some the games that were made then." For P12, experimenting and practicing similar development experiences before participating (e.g., watching videos of past jams or remaking similar games) was necessary to further strengthen his skills and be better prepared for his engagement in any potential game jams.

In particular, participants of both face-to-face and virtual game jams highlighted the importance of being comfortable with the development tools. P2 (26, male, Austria, hobbyist) said,

"I learned the possible engines I use. You need to freshen up on the experiences you had with the tool from the past and get yourself familiar again...you're to trust in whatever tool you intend to use."

According to him, the more familiar and comfortable he was with the development tool, the more confident he was when engaging in the development process in either virtual or face-to-face game jams. Similarly, P11 (18, male, India, hobbyist) also shared that he would make every tools that he needed ready in use to save time in a game jam: "I just made sure that I had all my software installed." And if it was allowed, it was additionally helpful and efficient to install necessary assets and engines before the game jam started, as P4 
(35, male, United States, hobbyist) noted:

"So normally I do have the platform I'm going to use ready, like if it's going to be Unity, like I have my blank project ready and I'm ready to go and load it up."

In summary, game developers who participated in either virtual or face-to-face game jams endeavored to make similar preparations regarding skills and tools, which would lay a solid foundation for them to better perform, engage, and experience in those jams.

Preference for Familiarity When Teaming. Obviously, different strategies for forming teams may emerge in virtual or face-to-face game jams due to their unique natures (e.g., computer-mediated methods in virtual game jams and in-person settings in face-to-face game jams). Yet, many participants expressed the same preference for teaming up with people they already knew regardless of the mode of game jams. P5 (28, male, Malaysia, hobbyist) explained that cultural background and personality might lead to such tendency: "Usually in Malaysia, we are kind shy so usually we stick to friends we know or coworkers no matter in online or offline jams."

P4 (35, male, United States, hobbyist) believed that the game jams being time-sensitive events also contributed to this choice:

"Yeah, most of the time I will be with people I know in either online or offline game jams. And it would be from just years of going to events with them, having boots next to them, and hanging out. So there's definitely a lot of long term good relationships that can be formed in those jams."

For him, teaming up with people whom he already knew significantly improved his experiences of game jams. In his opinion, it was much easier for teammates who were familiar with each other to reach common understandings and foster a more harmonious atmosphere for collaboration. This may also promote long-term and supportive social relationships.

In contrast, participants were worried that teaming up with strangers in either face-to-face or virtual game jams may lead to an unorganized development process. P7 (18, male, Canada, hobbyist) and P16 (30, female, United States, full-time indie) pointed out:

"I put out a message asking if anyone wanted to collaborate for the game jam, who was interested in the same theme as me. And he said, 'Yeah, sure.' So that's what we did for that one. I feel like we didn't communicate our differences very well. And I don't think that the work was divided amongst us as well as it could have been." (P7)

"I've only worked with a random team once, but that game jam didn't go anywhere. So usually, I work with people I already know." (P16)
For both participants, working with strangers seemed to cause reduced efficiency and much less ideal outcomes in the game jams due to the poor communication, misallocation of tasks, and the lack of mutual understanding of each teammate's strengths and work styles. Therefore, working with acquaintances or people whom they already knew appeared to be a preferred method in both virtual and face-to-face game jams, which not only helped accomplish the collaborative development goal but also supported a more positive social experience.

Struggles with Time Management. Participants of both virtual and face-to-face game jams also experienced the same challenge of time management in their highly intensive development process, as P6 summarized, "either online or offline, the biggest hurdle is timing." P2(26, male, Austria, hobbyist) further explained:

"I struggled with coming up with an idea based on the theme that was given to us.... It almost took the all first night but I finally did... Then we had to work faster, didn't get a lot of sleep throughout the jam."

According to P2, not knowing how to manage and allocate time effectively was a common experience for participants in any mode of game jams. Very often, they tended to spend too much time on a single task, which resulted in rushing to meet deadlines, disrupted plans, and a negative impact on the balance of work and breaks.

One reason why time management is such a challenging experience in both virtual and face-to-face game jams lies in the tension between reasonable time allocation and the desire for excellent results in the time-sensitive process of creation. P17 (25, male, Belgium, full-time indie) described:

"But at the same time, you don't want to jump right into the first idea because it might not be a very good idea. In which case, you're likely spend a lot of time working on it before you decide that there might have been something better."

According to P17, on the one hand, developers needed a sufficient amount of time to carefully evaluate the potentials of different ideas, which would directly lead to the success or failure of a game. Yet, on the other hand, they needed to decide when would be the right time to move on. How to balance this decision-making process, therefore, was a critical experience in any mode of game jams.

Another reason lies in game development as a complex creative process embedded with a series of parallel and interdependent tasks. P2 (26, male, Austria, hobbyist) shared:

"Deciding who does what is important for both online and offline game jams. You can make your fairly 
efficient workflow if you have those planned out well, because some tasks depend on other tasks to be finished, and some don't. So, maybe map out your critical path and see which elements you need before you start another task."

In P2's opinion, the goal of time management in any mode of game jams was to effectively compress, organize, and prioritize activities for maximum efficiency. In this sense, accurately identifying and allocating tasks for each team member played an essential role in time management. However, as the development process proceeded, it was difficult to maintain the original time/task distribution scheme due to the dynamic and creative nature of game development, which made time management in game jams even more challenging.

Challenges for Identifying the Appropriate Scope of Work. Another common experience that participants of both virtual and face-to-face game jams mentioned was the challenge of getting an idea of the right scope" (P2, 26, male, Austria,hobbyist). P9 (50, male, United States, full-time indie) described his experience in virtual game jams:

"Over-scoping is the big challenge in game jams. The number one easy thing to do is to try and do too much and then have almost nothing to show. Because everything is 50\% done instead of one thing being $100 \%$ done and in great shape."

P4 (35, male, United States, hobbyist) shared similar experiences in face-to-face game jams:

"The biggest thing is always going to be scope creep and not completing. A huge percentage of games made in game jams are unfinished and almost unplayable sometimes."

According to these quotes, game jams participants, regardless of virtual or face-to-face, often tended to go with the flow and bring forward many new ideas for developing creative games. However, without an adequate understanding of the scope of their development process and outcome, they would easily be distracted and hardly make any substantial progress. As a result, it was challenging for them to generate complete and desirable outcomes (i.e., good quality and playable games) within a limited time frame.

In particular, even when participants were well aware of the challenge of scoping, it was difficult to define the appropriate scope of a given project clearly. P2 (26, male, Austria, hobbyist) highlighted how keeping the scope of work from increasing remained the main obstacle in any mode of game jams:

"I still make the mistake regarding scope from time and time. It is simply too hard to control the scope-any of our ideas is too big for two days or a few days."
Therefore, it all comes down to a balance between one's aspiration or ambition for creativity and innovation and what one can actually achieve with team members in a limited time frame. P2's suggestion was to define this balance at the very beginning of the development process so as to make a plan of a reasonable scope: "So you should plan for a game that you can reason, that you can finish in a reasonable amount of time [...] Just cut down on the idea."

Another suggestion was to envision the potential game "as small as possible," as P4 (35, male, United States, hobbyist) noted: "So I think starting as small as possible, and then solving that to the point where you can add to is normally a pretty good strategy."

In P4's opinion, starting from "small" followed by add-ons depending on the actual development process and capacity was effective to avoid over scoping in game jams. This strategy would not only help developers complete their tasks within a limited time but also keep the scope of the project under control throughout the process.

\subsection{Unique Aspects of Social Collaboration in Virtual vs. Face-to-Face Game Jams}

Despite the shared experiences across virtual and face-to-face game jams, we also found that different modes of game jams afforded social collaboration in different means in terms of flexibility, social pressure, and richness of social interaction.

Flexibility. Our data show that virtual game jams provided game developers with more autonomy and freedom due to the absence of physical presence. P24 (36, female, United States, full-time indie) shared:

"I prefer online. I think that's mostly because I live with my husband, who I also collaborate with. So it's much more convenient for us to just be here. And we have all our equipment here and everything. I think if we sometimes have people over and we can even have a game jam at our house."

For P24, participating in virtual game jams was, in fact, an advantage over any face-to-face jams - she already had all the required resources at home, which offered a more coherent and comfortable atmosphere for her to engage in game development.

Other participants pointed out how it was more efficient and practical for them to participate in virtual game jams because they broke the geographical and temporal constraints. For example, P3 (24, female, unknown location, hobbyist) and P11 (18, male, India, hobbyist) explained:

"You can kind of go ahead and work on it whenever. It is very helpful for me because I have a full time job." 
(P3)

"The day when the virtual game jam started was also the day when I had my physical education test. But it was a 48 hour online thing so it was quite manageable." (P11)

For P3 and P11, engaging in virtual game jams was more enjoyable than face-to-face jams because they were able to manage their time more flexibly. In doing so, they not only ensured their participation in game jams but also maintained a normal daily life during the jam.

In addition, participating in virtual game jams seemed to help participants better manage the balance between work and rest. For example, P23 (24, male, England, hobbyist) described how the freedom of time control and deployment in virtual game jams helped him keep a healthy sleeping and eating schedule:

"I tend to do my own thing a bit more and make sure I get a decent amount of sleep and eat properly during game jams. I tend to allocate myself a little more time to look after myself and make sure I'm actually mentally awake. You can easily do this during a virtual game jam."

In contrast, in face-to-face game jams, participants were worried that the more open and public the environment was, the more likely others would scrutinize their behavior, and the less flexibility they would have. P23 shared: "In person game jams were very kind of public and everybody knew it was happening. You probably could not sleep or really be yourself." According to P23, face-to-face game jams were public events where everyone could observe what others were doing. Such social presence often created peer pressure, making participants more likely to stress out about their behaviors and self-images.

In some sense, face-to-face game jams also foster a more intense and exhausting social atmosphere for game development. P18 (51, male, Russia, full-time indie) mentioned how extra efforts were often required in face-to-face game jams to keep developers focusing on the tasks:

"People are at times lacking sleep [...] we had a friend who participated was no skill related to video games, but she took care of everything like, like buying pizza, bringing them and stuff like that."

P5 (28, male, Malaysia, hobbyist) also mentioned that giving up sleep and rest to go with the vibe and workflow was a common experience in face-to-face game jams:

"Once entered, usually the announcer will give us a theme to follow. Thus, from there onward we improvise through hours and sometimes through day and night [...] I usually have energy problems in game jams."
Therefore, for these developers, virtual game jams often afforded more relaxing and flexible experiences of engaging in game development, whereas face-to-face game jams tended to be more intense and demanding.

Social Pressure. A high degree of flexibility may also lead to a loose sense of teamwork and responsibility. Participants revealed that while virtual game jams were more flexible, they sometimes encouraged less social responsibilities. One potential reason may be that it is easier for people to avoid complaints and blames from their teammates in a virtual game jam without physical presence. For example, P23 (24, male, England, hobbyist) believed that there was much less pressure and no psychological burden to give up in virtual game jams: "While online, it's quite easy to just saying I'm done for the night. I'm going to bed." Therefore, a sense of self-control and self-supervision is highly important for engaging in virtual game jams, which would help developers stay motivated and fully assuming their responsibilities even without their teammates and competitors' social presence.

In contrast, face-to-face game jams effectively foster and promote a social atmosphere of hard-working, collaboration, and responsibilities. P22 (22, male, United States, hobbyist) highly praised face-to-face game jams as they directly inspired every participant on site:

"I think the face-to-face ones are definitely more impactful and meaningful. You get to see how people work on games together."

For P22, physical presence was one of the most beneficial experiences of participating in face-to-face game jams. Participants not only directly learned from one another but also built a stronger sense of community - "see[ing] how people work on game together." P23 (24, male, England, hobbyist) also highlighted how engaging in face-to-face game jams motivated participants to perform better and create high quality products:

"In face-to-face game jams, you don't want to let everybody else down. So you don't want to sleep and you don't want to see them make more mistakes. So I guess the in person, there's a bit more pressure to do well."

In this sense, the more competitive and encouraging the situation developers were in, the more likely they would feel guilty about giving up, especially in a face-to-face setting with teammates. Therefore, participants tended to be more socially responsible in face-to-face game jams where they could directly witness and experience everyone's behaviors and emotions such as excitement, tension, and anxiety. 
The Richness of Social Interaction. Compared to those in face-to-face game jams, participants in virtual game jams appeared to engage in less social interaction. In general, they tended to heavily depend on text chat rather than any other mode of communication. P1 (30, male, United States, hobbyist) and P3 (24, female, location unknown, hobbyist) shared:

"We haven't been able to find a better tool than Discord to communicate. I would say like maybe 80 to $90 \%$ of our communication is text." (P1)

"We use a Discord channel. So it was nice because we could message people and voice call them if we needed to, it was more text and then occasional voice." (P3)

Participants were well aware that text chat did not afford the exchange of social and emotional cues or provide contextual information of their communication. Yet they were still reluctant to use voice or video chat in a virtual game jam, as P3 shared:

"My team members didn't want to communicate over. They didn't want to talk. So it was a lot of typing and sending pictures."

Therefore, a significant advantage of face-to-face game jams over virtual game jams is that they afford much richer social interaction. P2 (26, male, Austria, hobbyist) described:

"The positive part of face-to-face game jams is that everyone is physically there and the communication is here. So you can show them what is happening on screen and what you expect. If there is miscommunication, you can identify it faster."

According to P2, communication and interaction in a face-to-face game jam could happen immediately at the moment without any delay. In this way, not only the collaboration would be more straightforward and efficient but also the social experience could be more positive - as less miscommunication would occur.

In this sense, some participants considered that virtual game jams tended to be more technical while face-to-face game jams focused on sociability:

"I think that in a virtual game jams people tend to close themselves up a little bit and it's much more technical." (P13, 24, male, Brazil, full-time indie)

"It is always good to wander around to speak with everyone, to know to see what people are making. This social part is always really fun." (P18, 51, male, Russia, full-time indie)

In P13's and P18's accounts, without direct physical interaction, virtual game jams appeared to be less sociable and show more restrained interaction (e.g., "people tend to close themselves up a little bit". Yet socializing was considered an essential experience of face-to-face game jams, either for teaming, collaborating, or just hanging out together.

In summary, virtual and face-to-face game jams tend to afford varying degrees of social interaction and engagement. However, participants valued both modes, as they articulated creative flexibility and intensive interaction respectively - both necessary components of game development.

\section{Discussion}

To answer our research question, we have highlighted: 1) regardless of engaging in virtual or face-to-face game jams, game developers shared common experiences including the need to be prepared in terms of skills, tools, and software installation; the preference for familiarity when teaming; and struggles with time management and scope of work; 2) different modes of game jams also afforded social collaboration in different means in terms of flexibility, social pressure, and richness of social interaction.

In this section, we discuss the implications of our findings for better understanding the differences between virtual and face-to-face game jams and their affordances for game developers' social collaboration.

\subsection{Game Jams as Novel Social Collaboration Spaces}

In our study, participants regarded game jams as valuable sites for social collaboration in game design and development, which formed a unique culture of innovation. Similar to makerspaces or hackerspaces, game jams focus on collaborative exploration, creation, and hands-on learning [28]; they also provide more opportunities for the general public (e.g., non-professional developers or hobbyists), rather than tech professionals, to engage in innovation $[29,30]$.

However, compared to other similar social collaboration spaces such as makerspaces, hackerspaces, or fablabs, both virtual and face-to-face game jams offer unique social and technological dynamics for game developers' collaboration. First, game jams are the intrinsically time-sensitive and intense process of innovation. This highlights the role of uncertainty (e.g., the randomness of teammates and the unpredictability of themes) in developers' experiences of game jams. Many participants appreciated this aspect and considered working under time pressure a necessary part of most game jam experiences. In addition, engaging in game jams emphasizes the core value of creativity and playability rather than the goal of creating a complete and polished game within a short time frame. This emphasis directly affects how game developers perceive and experience game jams 
- the collaborative exploration and innovation of core gameplay and rapid implementation.

Second, game jams seem to involve more complex and multidimensional interaction dynamics, including programming, storytelling, interactive design, music, art design, and presentation. In this sense, team members in game jam are required to interact in a manner that centers on the transparency of individual progress, timeliness of communication, and high efficiency of collaboration. Compared to other social collaboration spaces, collaborative experiences in game jams appear to be more intense and critical to successfully complete a project.

Third, the freedom for creativity and innovation embedded in game jams provides game developers with both more challenging and promising community engagement. On the one hand, developers experienced more pressure on social interaction due to the difficulties of managing time and identifying the appropriate scope of work, the randomness of forming teams, and the need to collaborat with strangers in an intensive way. On the other hand, they also enjoyed such experiences of working with people with diverse backgrounds in a highly efficient way, which exposes them to diverse opinions and perspectives and opens up more opportunities for creativity and innovation together.

Therefore, similar to fablabs or makerspaces, game jams promote game development as civic efforts [31, $22,32]$ and provide game developers with spaces for collaborative production and community building [33, $34,35]$. They also emerge as unique social collaboration spaces as they highlight time-sensitive and intensive teamwork, involve more complex and multidimensional interaction dynamics, and afford more challenging and promising community engagement.

\subsection{The Role of Different Modes of Game Jams in Game Development}

As our findings suggest, game jams could offer several social and technological benefits to the game design and development community. Such advantages were also commonly found in other forms of tech events such as hackathons. For example, we observed similarities between our findings and prior work on face-to-face and virtual hackathons. In both hackathons and game jams, familiarity of teammates and preparation of tools were noted to be important to attendees [23]. Additionally, compared to online/virtual events, face-to-face events demonstrate notable benefits for interpersonal communication and teamwork due to co-location [23].

Yet our findings also highlight the unique roles of different modes of game jams in facilitating and supporting game development. In our findings, face-to-face game jams in some ways significantly cultivate a sense of community. Such events place more focus on face-to-face interactions and communications, which benefits participants and the game development community in a variety of ways, including having mentors, more engaging in the development environment, and developing for a client [36]. In particular, game jams have a more specific set of preferences than general hackathons, which functions as a more cohesive community of practice - for example, people who share similar interests in game design or development will come together to collaborate or compete. In fact, the focus on community, networking, and learning are some of the most important reasons for attending game jams [37]. In this sense, face-to-face game jams are valued differently from online/virtual events - its focus on face-to-face development revolves around interpersonal communication and in-person opportunities specific to a particular game jam.

However, face-to-face game jams often tend to fall short of supporting flexibility and freedom in game development, which points to a key challenge in game development - accessibility. While game jams are intended to be open to anyone who is interested in game development, these events are traditionally specific to a specific region and demographic. As a result, only a particular group of people can attend - those who can afford the cost of time, effort, and travel. Our findings demonstrate that virtual game jams improve the accessibility of game development by allowing for the flexibility of location, work-related responsibilities, and general wellness - all of which may not be easily facilitated in face-to-face game jams. In particular, despite through computer-mediated communication methods, participants of virtual game jams value the ability to still feel connected to the community.

In summary, we have shown that despite their different advantages and disadvantages, both face-to-face and virtual game jams support game developers' social collaboration to innovate game design and development. Yet game developers also value them in different ways. In face-to-face game jams, participants value networking, collaboration, and the interpersonal aspects of game development. In virtual game jams, they value the flexibility, freedom, and accessibility while still maintaining the feeling of being social and connected in the broader game development community. 


\subsection{Limitations}

In this study, we focus on comparing virtual and face-to-face game jams for social collaboration that aim at indie game developers and hobbyists. These findings may not represent how other types of game jams, such as those focusing on industrial partners and educational purposes. In addition, the majority of our sample is male, with many being hobbyists. Future work is needed to recruit a more diverse sample in terms of age and gender to further verify the study's findings.

\section{Conclusions}

Game jams are virtual or face-to-face events that focus on agile game development. To participate in such events, game developers have a set time frame to build a digital game that fits the theme of the given event. Increasingly, game jams, regardless of virtual or face-to-face, have attracted a wide range of non-professional and hobbyist game developers to engage in game development collaboratively. As these events continue to grow in popularity, important questions emerge regarding how they shape game development by affording social collaboration. Our comparative study has identified the common experiences of both face-to-face and virtual game jams as well as their key differences. Our findings suggest that participants in virtual and face-to-face game jams shared similar experiences in terms of preparation regarding skills, tools, and software; the preference for familiarity when teaming; and challenges with the scope of work and time management. However, we also identified some notable differences between virtual and face-to-face game jams regarding flexibility, social pressure, and richness of social interaction involved in game developers' social collaboration. As global events (e.g., COVID-19) have placed more focus on understanding and re-imagining virtual collaboration, we hope that these findings not only shed light on the unique roles and values of face-to-face and virtual game jams in innovating game development but also highlight the underlying social and technological dynamics for successful computer-mediated collaboration.

\section{Acknowledgments}

We thank our participants and the anonymous reviewers. We also thank Jake Armstrong for helping with data collection and Nathan McNeese for his useful suggestions for improving this paper. This work was supported by the National Science Foundation under award 1849718.

\section{References}

[1] A. Kultima, "Defining game jam.," in FDG, 2015.

[2] IGJ0, "Indie game jam." http: / / indiegamejam. $\mathrm{com} / \mathrm{igj0/.}$

[3] S. Chen, "A brief history of game jams," Gamasutra, 2017.

[4] A. Zook and M. O. Riedl, "Game conceptualization and development processes in the global game jam," in Workshop proceedings of the 8th international conference on the foundations of digital games, pp. 1-5, 2013.

[5] A. Fowler, F. Khosmood, A. Arya, and G. Lai, "The global game jam for teaching and learning," in Proccedings of the 4th Annual Conference on Computing and Information Technology Research and Education New Zealand, pp. 28-34, sn, 2013.

[6] T. Faas, I.-c. Liu, L. Dombrowski, and A. D. Miller, "Jam today, jam tomorrow: Learning in online game jams," Proceedings of the ACM on Human-Computer Interaction, vol. 3, no. GROUP, pp. 1-27, 2019.

[7] J. Pirker, D. Economou, and C. Gütl, "Interdisciplinary and international game projects for creative learning," in Proceedings of the 2016 ACM Conference on Innovation and Technology in Computer Science Education, pp. 29-34, 2016.

[8] G. Freeman, J. Bardzell, S. Bardzell, and N. J. McNeese, "The innovation ecology: Collaborative information, community support, and policy in a creative technology community," in International Conference on Information, pp. 614-624, Springer, 2019.

[9] G. Freeman, N. McNeese, J. Bardzell, and S. Bardzell, “" pro-amateur"-driven technological innovation: Participation and challenges in indie game development," Proceedings of the ACM on Human-Computer Interaction, vol. 4, no. GROUP, pp. 1-22, 2020.

[10] G. Freeman and N. J. McNeese, "Exploring indie game development: Team practices and social experiences in a creativity-centric technology community," Computer Supported Cooperative Work (CSCW), vol. 28, no. 3-4, pp. 723-748, 2019.

[11] G. Freeman, J. Bardzell, S. Bardzell, and N. McNeese, "Mitigating exploitation: Indie game developers' reconfigurations of labor in technology," Proc. ACM Hum.-Comput. Interact., vol. 4, May 2020.

[12] W. Goddard, R. Byrne, and F. Mueller, "Playful game jams: guidelines for designed outcomes," in Proceedings of the 2014 Conference on Interactive Entertainment, pp. 1-10, 2014.

[13] A. Fowler, F. Khosmood, and A. Arya, "The evolution and significance of the global game jam," in Proc. of the Foundations of Digital Games Conference, vol. 2013, 2013.

[14] A. Fowler, J. Pirker, I. Pollock, B. C. de Paula, M. E. Echeveste, and M. J. Gómez, "Understanding the benefits of game jams: Exploring the potential for engaging young learners in stem," in Proceedings of the 2016 ITiCSE Working Group Reports, pp. 119-135, 2016.

[15] S. R. Yamane, "Adaptability of the global game jam: A case study in japan," in Proceedings of the 8th International Conference on the Foundations of Digital Games, 2013. 
[16] K. Shin, K. Kaneko, Y. Matsui, K. Mikami, M. Nagaku, T. Nakabayashi, K. Ono, and S. R. Yamane, "Localizing global game jam: Designing game development for collaborative learning in the social context," in International Conference on Advances in Computer Entertainment Technology, pp. 117-132, Springer, 2012.

[17] J. Pirker, A. Kultima, and C. Gütl, "The value of game prototyping projects for students and industry," in Proceedings of the International Conference on Game Jams, Hackathons, and Game Creation Events, pp. 54-57, 2016.

[18] J. A. Preston, J. Chastine, C. O’Donnell, T. Tseng, and B. MacIntyre, "Game jams: Community, motivations, and learning among jammers," International Journal of Game-Based Learning (IJGBL), vol. 2, no. 3, pp. 51-70, 2012.

[19] R. Ramzan and A. Reid, "The importance of game jams in serious games," in 10th European Conference on Games Based Learning: ECGBL 2016, pp. 538-546, Academic Conferences and Publishing International Ltd.(ACPI), 2016.

[20] P. Blikstein and D. Krannich, "The makers' movement and fablabs in education: experiences, technologies, and research," in Proceedings of the 12th international conference on interaction design and children, pp. 613-616, 2013.

[21] G. Briscoe, "Digital innovation: The hackathon phenomenon," 2014.

[22] N. Taylor, U. Hurley, and P. Connolly, "Making community: the wider role of makerspaces in public life," in Proceedings of the 2016 CHI Conference on Human Factors in Computing Systems, pp. 1415-1425, 2016.

[23] E. H. Trainer, A. Kalyanasundaram, C. Chaihirunkarn, and J. D. Herbsleb, "How to hackathon: Socio-technical tradeoffs in brief, intensive collocation," in proceedings of the 19th ACM conference on computer-supported cooperative work \& social computing, pp. 1118-1130, 2016.

[24] M. Raatikainen, M. Komssi, V. Dal Bianco, K. Kindstöm, and J. Järvinen, "Industrial experiences of organizing a hackathon to assess a device-centric cloud ecosystem," in 2013 IEEE 37th Annual Computer Software and Applications Conference, pp. 790-799, IEEE, 2013.

[25] S. Almishari, N. Salamah, M. Alwan, N. Alkhalifa, and A. Al-Wabil, "The rise of hackathon-led innovation in the mena region: Visualizing spatial and temporal dynamics of time-bounded events," in International Conference on Social Computing and Social Media, pp. 367-377, Springer, 2017.

[26] L. Dabbish, C. Stuart, J. Tsay, and J. Herbsleb, "Social coding in github: transparency and collaboration in an open software repository," in Proceedings of the ACM 2012 conference on computer supported cooperative work, pp. 1277-1286, 2012.

[27] A. L. Strauss, Qualitative analysis for social scientists. Cambridge university press, 1987.

[28] C. R. Forest, R. A. Moore, A. S. Jariwala, B. B. Fasse, J. Linsey, W. Newstetter, P. Ngo, and C. Quintero, "The invention studio: A university maker space and culture.," Advances in Engineering Education, vol. 4, no. 2, p. n2, 2014.
[29] A. C. Barton, E. Tan, and D. Greenberg, "The makerspace movement: Sites of possibilities for equitable opportunities to engage underrepresented youth in stem," Teachers College Record, vol. 119, no. 6, pp. 11-44, 2016.

[30] R. S. Kurti, D. L. Kurti, and L. Fleming, "The philosophy of educational makerspaces part 1 of making an educational makerspace," Teacher Librarian, vol. 41, no. 5 , p. 8, 2014.

[31] J. S. Hui and E. M. Gerber, "Developing makerspaces as sites of entrepreneurship," in Proceedings of the 2017 ACM Conference on Computer Supported Cooperative Work and Social Computing, pp. 2023-2038, 2017.

[32] A. L. Toombs, S. Bardzell, and J. Bardzell, "The proper care and feeding of hackerspaces: Care ethics and cultures of making," in Proceedings of the 33rd annual ACM conference on human factors in computing systems, pp. 629-638, 2015.

[33] A. Wiggins, D. Gurzick, S. Goggins, and B. Butler, "Quality hackathon: Evaluating the products of online co-production systems," in Proceedings of the 18th International Conference on Supporting Group Work, pp. 321-323, 2014.

[34] P. Johnson and P. Robinson, "Civic hackathons: Innovation, procurement, or civic engagement?," Review of policy research, vol. 31, no. 4, pp. 349-357, 2014.

[35] E. H. Trainer, C. Chaihirunkarn, A. Kalyanasundaram, and J. D. Herbsleb, "Community code engagements: summer of code \& hackathons for community building in scientific software," in Proceedings of the 18th International Conference on Supporting Group Work, pp. 111-121, 2014.

[36] M. Lara and K. Lockwood, "Hackathons as community-based learning: a case study," TechTrends, vol. 60, no. 5, pp. 486-495, 2016.

[37] A. Fowler, "Informal stem learning in game jams, hackathons and game creation events," in Proceedings of the International Conference on Game Jams, Hackathons, and Game Creation Events, pp. 38-41, 2016. 